\title{
Beyond Crystallography: Diffractive Imaging Using Coherent X-ray Light Sources
}

\author{
Jianwei Miao $^{1 *}$, Tetsuya Ishikawa ${ }^{2}$, Ian K. Robinson ${ }^{3,4}$ \& Margaret M. Murnane ${ }^{5}$ \\ ${ }^{1}$ Department of Physics \& Astronomy and California NanoSystems Institute, University of \\ California, Los Angeles, CA 90095, USA. \\ ${ }^{2}$ RIKEN SPring-8 Center, Kouto 1-1-1, Sayo, Hyogo 679-5148, Japan. \\ ${ }^{3}$ London Centre for Nanotechnology, University College London, 17-19 Gordon Street, WC1H \\ $0 \mathrm{AH}, \mathrm{UK}$. \\ ${ }^{4}$ Research Complex at Harwell, Harwell Campus, Didcot, Oxford OX11 0DE, UK. \\ ${ }^{5}$ JILA, University of Colorado at Boulder, Boulder, CO 80309, USA. \\ *Corresponding author. E-mail: miao@physics.ucla.edu
}

\begin{abstract}
X-ray crystallography has been central to the development of many fields of science over the past century. It has now matured to a point that as long as good quality crystals are available, their atomic structure can be routinely determined in three dimensions. However, many samples in physics, chemistry, materials science, nanoscience, geology, and biology are non-crystalline and thus their three-dimensional structures are not accessible by traditional $\mathrm{x}$-ray crystallography. Overcoming this hurdle has required the development of new coherent imaging methods to harness new coherent x-ray light sources. Here we review the revolutionary advances that are transforming x-ray sources and imaging in the $21^{\text {st }}$ century.
\end{abstract}


The x-ray science community has witnessed two revolutionary developments over the past two decades. First, large- and small-scale coherent x-ray sources, including advanced synchrotron sources, x-ray free electron lasers (XFELs) and high harmonic generation (HHG) sources, are under rapid development worldwide (1-3). Compared with the previous generations of x-ray sources, the new light sources have remarkable properties. XFELs increase the coherent x-ray flux by nine orders of magnitude, while tabletop HHG sources generate a coherent x-ray supercontinuum that spans the entire electromagnetic spectrum from the UV to the $\mathrm{keV}$ region, covering 12 octives of bandwidth. In addition, HHG and XFEL pulses are extremely short, ranging from tens of attoseconds to hundreds of femtoseconds. Second, a new approach to x-ray crystallography, known as coherent diffractive imaging (CDI), enables structure determination of non-crystalline specimens and nanocrystals with a resolution limited only by the spatial frequency of the diffracted waves (4). Moreover, CDI enables simultaneous amplitude and phase contrast imaging. In recent years, the combination of powerful coherent x-ray light sources and CDI methods, coupled with advanced x-ray detectors and computational algorithms, has opened up new research frontiers in the physical and biological sciences that are simply not attainable using conventional x-ray crystallography (5-19). Here, we review progress in CDI and the advanced x-ray light sources that are allowing this new imaging modality to blossom. We highlight the exciting scientific discoveries that were enabled by these new capabilities, and look forward with great anticipation to the new frontiers in imaging that are emerging.

\section{Coherent Diffractive Imaging Methods}

In a traditional microscope, a lens is used to collect the light scattered by a sample and thereby recover an image. However, in the x-ray region, the use of lenses introduces a severe limitation because diffractive x-ray optics must be used, and these are far from perfect. Although x-ray 
microscopes with $\sim 10 \mathrm{~nm}$ spatial resolution have been demonstrated, $\sim 25 \mathrm{~nm}$ is more typical which is nowhere near the wavelength limit (20). CDI avoids this limitation by illuminating an object with a coherent laser-like beam, and collecting the scattered light on a detector (Figs. 1AE). The image of the object can then be recovered using an advanced phase retrieval algorithm by taking advantage of the fact that the diffracted wave is proportional to the Fourier transform of the object (4). However, while the magnitude squared of the Fourier transform can be measured by a detector, the phase information is lost. This constitutes the well-known phase problem associated with recovering an image from a diffraction pattern. For a non-crystalline specimen, the diffraction pattern is continuous and can be sampled at a frequency finer than the Nyquist interval (the inverse of the specimen size). For a noise-free diffraction pattern, when the number of independently sampled intensity points is larger than (or equal to) the number of unknown variables associated with a specimen, the phase information is in principle encoded inside the diffraction intensity (21) and can be usually retrieved by iterative algorithms $(22,23)$. Furthermore, because no optics is inserted between the specimen and detector, CDI represents the most photon-efficient $x$-ray imaging modality (24).

\section{Iterative phase retrieval algorithms}

A common feature of these algorithms requires iterating between real and reciprocal space (23), consisting of the following four steps in each iteration (Fig. 1F): (i) the algorithms usually start with a random phase set as the initial guess. By combining this random phase set with the measured Fourier magnitude and then applying an inverse fast Fourier transform (FFT), an initial image is computed; (ii) depending on oversampling of the diffraction intensity, a support (i.e. a boundary slightly larger than the sample envelope) can be estimated from the image. The electron density outside the support of the image is reduced, while the negative electron density inside the support is modified, for example by setting to zero, depending on the algorithm; (iii) by applying a FFT to the updated image, a new Fourier transform is generated; (iv) by replacing its magnitude with the measured data, a better estimate of the Fourier transform is obtained and used for the next iteration. This process is then repeated, and each iteration is characterized by an error metric, defined as the difference between the calculated and measured Fourier magnitudes. Usually, after hundreds to thousands of iterations, the correct phase set can be retrieved. 
By combining phase retrieval algorithms (Text Box) and coherent x-ray sources, several CDI methods have now been developed in both transmission and reflection modes, including plane-wave CDI (PCDI), Bragg CDI (BCDI), ptychographic CDI (ptychography), Fresnel CDI, reflection CDI, sparsity CDI and others $(5-18,25,26)$. Figure 1A-E shows the schematic layout of the five major CDI methods to date. In the original PCDI, a plane wave illuminates an object in transmission, and the diffraction pattern is measured by an area detector (Fig. 1A). In order to satisfy the oversampling criterion, either an isolated object or a finite illumination had to be implemented. To obtain 3D structural information, a tilt series of diffraction patterns is acquired over various sample orientations and then phased to obtain a 3D image $(6,11)$. Because planewave $\mathrm{CDI}$ is insensitive to sample drift and vibration, it achieved the highest $2 \mathrm{D}$ and $3 \mathrm{D}$ spatial resolution $(\approx 2 \mathrm{~nm}$ and $5.5 \mathrm{~nm}$, respectively) of any x-ray imaging method $(27,28)$. In BCDI, a coherent beam of x-rays illuminates a nanocrystal and the diffraction pattern surrounding a Bragg peak is measured (Fig. 1B) $(5,8)$. Inversion of the diffraction pattern yields a complex 3D image, whose phase is related to the displacement and strain field of the crystal lattice. Furthermore, BCDI is also useful for powder and polycrystalline samples where many randomly oriented grains are in the beam at the same time. Because the Bragg peak of each grain will be in a different location in reciprocal space, individual grains can be isolated from their neighbours.

Ptychography brings powerful new imaging capabilities because it can image a extended sample in either reflection or transmission modes, with near-wavelength spatial resolution $(\approx 1.3 \lambda)$ horizontally, and sub-nm vertically $(18,29,30)$. In ptychography, the sample is scanned on a $2 \mathrm{D}$ grid to collect a series of diffraction patterns from partially overlapping regions (Fig. 1C). This additional redundancy in the diffraction data enables robust image reconstruction with quantitative phase contrast (12), while also extracting the complex x-ray illumination beam, 
correcting for errors in the scanning stages, and improving the convergence of the iterative phase retrieval process $(13,18)$. In Fresnel CDI, a sample is illuminated by a curved wavefront through defocusing of an x-ray beam and the resulting Fresnel diffraction pattern is measured by a detector (Fig. 1D). If the incident beam is independently characterized and the geometrical dimensions of the experiment are accurately determined, the exit wave at the sample can be quantitatively reconstructed by using an iterative algorithm with fast convergence (10).

Very recently, CDI has been successfully implemented in reflection mode, giving beautiful 3D height maps of a surface, with near-wavelength transverse resolution, sub-nm vertical resolution, and very high phase and amplitude contrast due to the short wavelength of the illumination light $(18,26,29,31)$. Moreover, the geometry is very simple: a coherent $\mathrm{x}$-ray beam illuminates a sample and the diffracted light can then be collected with very high numerical aperture (Fig. 1E). When combined with powerful ptychographic imaging, high quality images of extended samples can be obtained $(18,29)$. Reflection CDI is complementary to other imaging modalities such as scanning electron microscopy (SEM) and atomic force microcopy (AFM), allowing for long $>3 \mathrm{~cm}$ working distances, less damage, and higher contrast imaging than other approaches.

\section{A Revolution in Coherent X-ray Light Sources}

In the past decade there has also been a revolution in the development of both large-scale and tabletop coherent x-ray sources (Fig. 2). Large-scale x-ray sources are based on high-energy accelerators, which emit x-rays with varying degree of coherence (23). When the first so-called $3^{\text {rd }}$ generation $x$-ray synchrotron facilities were designed, it was widely believed that undulators

for hard x-rays would require high-energy electron beams. As a result, the first $3^{\text {rd }}$ generation facilities used electron storage rings with energies of 6-8 Gigaelectronvolt $(\mathrm{GeV})$, and low 
emittance electron beams to enhance the brightness of the undulator radiation (23). However, despite their small source size and long source-sample distance, these $3^{\text {rd }}$ generation facilities radiated only a small amount of spatially coherent light $(\approx 0.1 \%$ of total $)$. Nevertheless, by using a small aperture, this small fraction of spatially coherent light could be extracted and used to perform the first CDI experiment at the National Synchrotron Light Source (4). A maturation of undulator technology enabled the development of higher harmonics and shorter period undulators, which can produce hard x-rays without requiring high energy storage rings. Thus, lower energy $(\sim 3 \mathrm{GeV})$, medium-sized storage rings are now used for hard x-ray sources where a wide variety of CDI activities are now in progress. Another new and important trend of accelerator-based advanced $\mathrm{x}$-ray sources is the use of a new magnetic lattice, known as a multibend-achromat, to more tightly focus the electron beam, further reduce its emittance and increase the $\mathrm{x}$-ray brilliance (23).

In the turn of the $21^{\text {st }}$ century, brighter XFEL sources were developed (23) based on selfamplified spontaneous emission (SASE) (Fig. 2A, inset) (1,2). When a low-emittance, highdensity and high-energy electron bunch is injected into a long undulator $(\sim 100 \mathrm{~m})$, it initially emits synchrotron radiation. The emitted photons travel at the speed of the light inside the undulator, which is only slightly faster than the velocity of the high-energy electron bunch. As a result, the radiation interacts back with the electron bunch. If the undulator is designed such that the interaction makes the faster electrons slower and slower electrons faster, the electron bunch density is periodically modulated by the radiation, a process called micro-bunching. The process exponentially increases the intensity of the emitted radiation as well as the interaction between the radiation and the electron bunch, introducing "gain" in the similar way as a conventional laser. At the end of the long undulator, the gain is saturated and an extremely intense XFEL 
pulse is produced. The peak brightness of the XFEL pulse is $10^{9}$ times higher than that of the most powerful $3^{\text {rd }}$ generation source (Fig. 2A), while their pulse duration ranges from tens to hundreds of femtoseconds, although with lower repetition rate and some timing jitter.

In parallel with advances in large-scale coherent $\mathrm{x}$-ray facilities, the same revolution that visible lasers underwent in the 1960s is now happening for tabletop coherent $\mathrm{x}$-ray sources. Interestingly, the first tabletop-scale coherent soft x-ray source was not based on creating a population-inversion to support laser action, but was rather based on nonlinear optics. In nonlinear optics, the high electric fields that are present in a focused laser beam can drive electrons in a highly irregular (anharmonic) motion that re-radiates harmonics of the driving laser light at much shorter wavelengths. In HHG, the laser field intensity must be sufficient to tunnel ionize the atoms used as the non-linear medium, by suppressing the Coulomb field that normally binds the electron to the ion. For the few-femtosecond time interval during which this is happening, the laser-driven quantum wave-function of the electron can radiate coherent high harmonic $\mathrm{x}$-rays. If $\mathrm{x}$-rays from a macroscopic number of atoms interfere constructively to generate a laser-like beam, in a process called phase matching, HHG can have spectacular temporal and spatial coherence. This is because of the ultra-precise timing relationship between the laser and x-ray fields that are synchronized to less than one attosecond.

Although its physical manifestation is very different, HHG can also be thought of as a coherent laser-driven version of the Röntgen x-ray tube. However, the full power of femtosecond lasers for manipulating the quantum wave-function of an electron is now being realized. By changing the color and polarization of the driving laser, the HHG spectrum, pulse duration and polarization can be controlled $(3,32,33)$, thus making it possible to generate $\mathrm{x}$-ray bursts with durations from tens of attoseconds to tens of femtoseconds, with a coherent bandwidth spanning 
12 octives, from $<100 \mathrm{meV}$ to $\approx 1 \mathrm{keV}$. One recent surprising finding is that longer wavelength mid-infrared lasers can generate shorter wavelength bright $\mathrm{x}$-ray beams. Using $4 \mu \mathrm{m}$ driving lasers for example, HHG emerges as a broad coherent super-continuum, spanning the entire electromagnetic spectrum from the ultraviolet (UV) to the soft $\mathrm{x}$-ray $\mathrm{keV}$ region of the spectrum, to wavelengths $<8 \AA$. Moreover, these $\mathrm{x}$-rays emerge as isolated attosecond bursts, that are predicted to scale to the sub-attosecond (i.e. zeptosecond) regime using longer wavelength lasers (33). In contrast, using intense UV driving lasers, HHG emerges as a series of bright narrowband peaks, with $\approx 10$ fs pulse duration. Finally, by manipulating the electron wave-function using bi-chromatic (two-color) circularly polarized counter-rotating laser beams, it is now possible to produce bright circularly polarized harmonics that complement the bright linearly polarized HHG beams available for 20 years (32).

The powerful quantum coherence of HHG makes it ideal for imaging the fastest dynamics relevant to function in atoms, molecules, nanosystems and materials, at multiple atomic sites simultaneously. HHG is complementary to the powerful XFEL machines, because it can be driven by a small scale $(\approx \mathrm{mJ}$ energy) laser at very high repetition rates $(1-100 \mathrm{kHz})$. Although the pulse energies are lower than those available at XFELs (nJ vs. mJ), the repetition rates are higher, and ideal for several exciting applications discussed below $(3,32-34,41,42)$.

\section{Multidisciplinary Science Enabled by Coherent X-ray Light Sources and Diffractive Imaging}

CDI is ideally suited for quantitative 3D characterization of materials at the nanoscale: $x$-rays have a larger penetration depth than electrons, so that destructive sample preparation can often be avoided. Moreover, by quantifying the incident and diffracted x-ray flux, CDI can extract the mass density and thus distinguish different phases in materials in three dimensions (35). 
Furthermore, the presence of core shell x-ray absorption provides chemical contrast, while their polarization can be exploited to provide magnetic contrast and molecular orientation. Thus, CDI enables nanoscale chemical, elemental and magnetic mapping of complex matter (29,36-38). Finally, the high temporal resolution of the new coherent x-ray sources such as HHG and XFELs already make it possible to capture the fastest charge, spin and lattice motions in matter, on multiple length and time scales.

In recent work, PCDI was used to quantitatively image a molten Fe-rich alloy and crystalline olivine sample, which was synthesized at $6 \mathrm{GPa}$ and $1800^{\circ} \mathrm{C}$ to mimic the conditions of Earth's upper mantle (35). Figure 3A shows the 3D morphology of the Fe-rich and Fe-S phases in an olivine matrix in which the molten Fe exhibits varied shapes and sizes. A histogram of the 3D mass density distribution of the sample indicates that the Fe-rich and Fe-S phases change continuously instead of abruptly (Fig. 3B) - due to local temperature, pressure, geometry, and microscopic percolation mechanisms, which are difficult to probe using other imaging techniques.

In other exciting developments, Figure 3C shows a ptychographic image of a partially delithiated nanoplate of $\mathrm{LiFePO}_{4}$, a material with broad potential application in electrochemical energy storage (38). By acquiring multiple images across the Fe L-shell x-ray absorption edge and using them as input to principal component and singular value decomposition analysis, a chemical composition image of the partially delithiated nanoplate is obtained, showing the distribution of two chemical species: $\mathrm{LiFePO}_{4}$ and $\mathrm{FePO}_{4}$ (Fig. 3D). The resolution of this chemical image $(\sim 18 \mathrm{~nm})$ is nearly an order of magnitude higher than the probe size $(150 \mathrm{~nm})$ and more than three times better than that of state-of-the-art scanning transmisison x-ray microscopy 
(20). These results suggest that the (de)lithiation reaction of $\mathrm{LiFePO}_{4}$ is two-phase, where both phases coexist in these sub-micrometer nanoplates.

BCDI's unique ability to image displacement (strain) fields in 3D also has widespread applications in materials science and nanoscience. For example, it was used to understand the structural principles of metal nanoparticles $(5,8)$ and to obtain quantitative 3D images of the full strain tensor when the projections from three or more non-coplanar Bragg peaks are combined to yield the full vectorial displacement field (39). XFELs also provide sufficient coherent x-ray flux in a single monochromatic femtosecond pulse to record a slice through the BCDI diffraction pattern of a nanocrystal. A pump-probe BCDI experiment was carried out on 300nm diameter Au nanocrystals (17). The nanocrystals were first excited by an 800nm Ti-sapphire infrared laser pulse and then followed by snapshot imaging of the excited state using XFEL BCDI. Provided both laser and x-ray pulses were kept below their damage thresholds, full 3D diffraction patterns could be built up as a function of delay time from the same nanocrystal. In this way movies were captured of the strain associated with whole crystal vibrations, with a 200ps period (Fig. 3E). Two simple dilation breathing modes and a previously unknown shear mode were identified (17).

Using tabletop HHG, full field coherent imaging in both reflection and transmission geometries has been demonstrated, with record spatial resolution of $22 \mathrm{~nm}$ (transverse) and $6 \AA$ (axial) $(40,18,29)$. Figure 3F shows a ptychographic image of a surface, with spatial resolution $<1.3 \lambda$ or $40 \mathrm{~nm}$ horizontally, $6 \AA$ vertically, and acquired with $<1$ min $\mathrm{x}$-ray exposure time $(18,29)$. Moreover, the phase contrast in CDI is enhanced due to the use of extreme ultraviolet $(\lambda=30 \mathrm{~nm})$ illumination. Compared with SEM or AFM, HHG-based CDI enables higher-contrast 
full field imaging with less sample damage, with long working distances $(\approx 3-10 \mathrm{~cm})$, automatic correction for imperfect scanning stages, and femtosecond time resolution $(\approx 10 \mathrm{fs})$.

Several discoveries have been made recently simply by monitoring the time-dependent HHG diffraction from a material after excitation by a laser pulse, to uncover which mechanisms are responsible for nanoscale energy, charge and spin transport $(19,41)$. One remarkable demonstration of this capability showed that the cooling rate for a nanometer-size heat source depends on its proximity to other sources, cooling more rapidly when spaced close together than when isolated. This finding represents fundamentally new materials science directly relevant to the design of future energy-efficient nanosystems $(19,42)$. Real-time imaging in 3D through opaque materials will represent a powerful capability for understanding functional nanosystems.

For biological applications, CDI is complementary to optical and electron microscopies in terms of spatial resolution, sample thickness, contrast mechanism and quantitative capability. By using novel imaging technologies and labelling techniques, super-resolution fluorescence microscopy can study dynamic processes in living cells at the tens of nanometre level, but requires labelling of specific molecules, and can accommodate limited sample thickness. To achieve considerably higher resolution, electron microscopy is the method of choice, but is limited to imaging thinner samples owing to the short penetration depth of electrons. Compared with super-resolution and electron microscopy, CDI has three unique features. First, due to the large penetration depth of x-rays, CDI can image whole biological cells without the need of sectioning. Second, CDI takes advantage of the phase shift (contrast) of the intrinsic density in biological specimens that enables quantitative 3D imaging of the entire contents of cells and cellular organelles using their natural contrast. Finally, by avoiding the use of lenses, the 
resolution of CDI is limited only by how high a scattering angle (spatial frequency) the sample diffracts the illuminating light.

Using $3^{\text {rd }}$ generation synchrotrons, CDI has been used to image whole cells, cellular organelles, viruses and biological materials, with spatial resolution down to $\sim 11 \mathrm{~nm}(7,43-48)$. As an example, Fig. 4A shows the 3D mass density distribution of a whole, unstained yeast spore cell in which the 3D structure of intercellular organelles are quantitatively identified (44). Figure $4 \mathrm{~B}$ and $\mathrm{C}$ shows the $3 \mathrm{D}$ image of an unstained human chromosome as well as the first ever coherent diffraction pattern measured from a single, unstained virus particle and its reconstructed structure $(46,47)$. CDI has also been applied to study biological composite materials, including nanoscale imaging of mineral crystals in bone at different stages of mineralization (48) and quantitative 3D measurements of the osteocyte lacunae and the connecting canaliculi in a bone matrix (Fig. 4D, 15).

Using XFELs, CDI has been applied to measure the diffraction patterns of biological samples using single extremely intense and short X-ray pulses before the onset of radiation damage (diffraction-before-destruction) $(9,49)$. Two important directions have been pursued. One is to perform diffractive imaging of live cells, cellular organelles and virus particles with a resolution down to $\sim 18 \mathrm{~nm}(16,50-53)$, which is presently limited by the x-ray flux density on the sample. Figure 4E shows two representative diffraction patterns of individual mimivirus particles measured with single LCLS pulses and the corresponding reconstructed structures $(16,50)$. The other uses nanometre- to micrometre-sized protein crystals to significantly increase the resolution of diffraction patterns from single XFEL pulses (Figs. 4F and G) (54). This method, known as serial femtosecond crystallography, has achieved a highest resolution of $1.6 \AA(55)$ and 
also been applied to the structure determination of proteins that are difficult to grow as large crystals, such as membrane proteins $(56,57)$.

\section{Looking forward}

Looking into the future for this 2015 Year of Light, we can already identify three research frontiers in imaging science and applications using small and large-scale coherent x-ray light sources. First, by taking advantage of the extremely short XFEL and HHG pulses, CDI is ideally suitable to probe functioning systems at the nanoscale - at multiple sites simultaneously. A new form of CDI - hyperspectral imaging based on ptychography (12) - makes it possible to retrieve an image at several different x-ray wavelengths simultaneously, encoding instantaneous charge and spin information. Recently, dynamic CDI has been applied to image coherent acoustic phonons in nanocrystals, nano-fabricated structures undergoing laser ablation, and standing surface acoustic wave in a piezoelectric substrate with spatial resolutions of tens of nanometers and temporal resolutions of picoseconds to $<10 \mathrm{fs}(17,34,41,58,59)$. With further improvement in experimental design, dynamic CDI should be able to achieve spatial resolutions $<10 \mathrm{~nm}$ and temporal resolutions of $10 \mathrm{fs}$. Such a powerful imaging technique with high spatialtemporal resolutions is expected to profoundly expand our understanding of a wide range of dynamic phenomena, ranging from phase transitions, charge transfer, transport, nucleation, melting, superheating, crack and shock formation to lattice and grain boundary dynamics as well as materials discovery of transient "hidden" phases of matter.

Second, as the wavelength of x-rays is on the order of the size of an atom, scientists have long dreamed of the development of atomic-resolution x-ray microscopy. However, the highest resolution presently attainable by any $\mathrm{x}$-ray imaging method is $\sim 2 \mathrm{~nm}$ in $2 \mathrm{D}$ and $\sim 5.5 \mathrm{~nm}$ in $3 \mathrm{D}$ $(27,28)$. With the rapid development of coherent light sources that promise to increase the x-ray 
brilliance by several orders of magnitude (Fig. 2A), CDI may achieve atomic resolution for radiation hard samples. Although electron microscopy can routinely image crystalline materials at atomic resolution, $x$-rays have several unique properties compared to electrons: (i) $x$-rays interact with the electron density rather than the Coulomb potential of an atom; (ii) dynamical scattering is usually negligible, allowing study of thick specimens; and (iii) the sample can be placed in an ambient environment. Thus, achieving atomic-resolution CDI is anticipated to find broad applications in both fundamental and applied science.

Finally, one of the ultimate goals of CDI is to achieve high-resolution Single Particle Imaging (SPI), for example of protein complexes without the need of crystallization. Using ultra intense and short XFEL pulses, coherent x-ray diffraction patterns can be collected from single protein complexes based on the diffraction-before-destruction scheme (49). These diffraction patterns can then be assembled into 3D patterns from which a 3D image follows after inversion (60-62). Numerical simulations show that with $\sim 10^{5}-10^{6}$ identical copies of large protein molecules, the 3D structure of the molecules can be determined at near atomic resolution (60). Recent XFEL experiments have demonstrated the feasibility of the SPI method but at much lower resolution (16,50-53). In order to improve the spatial resolution, the XFEL pulse intensity has to be increased by $2-3$ orders of the magnitude. Recent work has suggested that the combination of self-seeding (using x-rays from the first half of the undulator to seed the second half via a monochromator) and undulator tapering (a gradual reduction of the field strength of an undulator to preserve the resonance wavelength) can achieve a 100-fold increase of the XFEL peak power (23). The increase of the XFEL pulse flux, coupled with large dynamic-range detectors, could realize the potential of SPI at higher resolution.

\section{References and Notes}


1. P. Emma et al., Nat. Photonics 4, 641-647 (2010).

2. T. Ishikawa et al., Nat. Photonics 6, 540-544 (2012).

3. T. Popmintchev et al., Science 336, 1287-1291 (2012).

4. J. Miao, P. Charalambous, J. Kirz, D. Sayre, Nature 400, 342-344 (1999).

5. I. K. Robinson et al., Phys. Rev. Lett. 87, 195505 (2001).

6. J. Miao et al., Phys. Rev. Lett. 89, 088303 (2002).

7. J. Miao et al., Proc. Natl. Acad. Sci. U.S.A. 100, 110-112 (2003).

8. M. A. Pfeifer et al., Nature 442, 63-66 (2006).

9. H. N. Chapman et al., Nat. Phys. 2, 839-843 (2006).

10. G. J. Williams et al., Phys. Rev. Lett. 97, 025506 (2006).

11. H. N. Chapman et al., J. Opt. Soc. Am. A 23, 1179-1200 (2006).

12. J. M. Rodenburg et al., Phys. Rev. Lett. 98, 034801 (2007).

13. P. Thibault et al., Science 321, 379-382 (2008).

14. K. S. Raines et al., Nature 463, 214-217 (2010).

15. M. Dierolf et al., Nature 467, 436-439 (2010).

16. M. M. Seibert et al., Nature 470, 78-81 (2011).

17. J. N. Clark et al., Science 341, 56-59 (2013).

18. M. D. Seaberg et al., Optica 1, 39-44 (2014).

19. M. E. Siemens et al., Nat. Mater. 9, 26-30 (2010).

20. A. Sakdinawat, D. Attwood, Nat. Photonics 4, 840-848 (2010).

21. J. Miao, D. Sayre, H. N. Chapman, J. Opt. Soc. Am. A 15, 1662-1669 (1998).

22. J. R. Fienup, Opt. Lett. 3, 27-29 (1978).

23. See supplementary text in supplementary materials on Science Online. 
24. X. Huang et al., Opt. Express 17, 13541-13553 (2009).

25. A. Szameit et al., Nat. Mater. 11, 455-459 (2012).

26. T. Sun et al., Nat. Photonics 6, 586-590 (2012).

27. Y. Takahashi et al., Phys. Rev. B 82, 214102 (2010).

28. R. Xu et al., Nat. Commun. 5, 4061 (2014).

29. D. E. Adams et al, Laser Focus World, in press (2015).

30. A. M. Maiden, J. M. Rodenburg, Ultramicroscopy 109, 1256-62 (2009).

31. S. Roy et al., Nat. Photonics 5, 243-245 (2011).

32. O. Kfir et al., Nat. Photonics 9, 99-105 (2015).

33. C. Hernández-García et al., Phys. Rev. Lett. 111, 033002 (2013).

34. E. Turgut et al., Phys. Rev. Lett. 110, 197201 (2013).

35. H. Jiang et al., Phys. Rev. Lett. 110, 205501 (2013).

36. C. Song et al., Phys. Rev. Lett. 100, 025504 (2008).

37. A. Tripathi et al., Proc. Natl. Acad. Sci. U.S.A. 108, 13393-13398 (2011).

38. D. A. Shapiro et al., Nat. Photonics 8, 765-769 (2014).

39. M. C. Newton, S. J. Leake, R. Harder, I. K. Robinson, Nat. Mater. 9, 120-124 (2010).

40. M. D. Seaberg et al., Opt. Express 19, 22470-22479 (2011).

41. D. Rudolf et al., Nat. Commun. 3, 1037 (2012).

42. K. Hoogeboom-Pot et al., Proc. Natl. Acad. Sci. U.S.A., in press (2015).

43. D. Shapiro et al., Proc. Natl. Acad. Sci. U.S.A. 102, 15343-15346 (2005).

44. H. Jiang et al., Proc. Natl. Acad. Sci. U.S.A. 107, 11234-11239 (2010).

45. J. Nelson et al., Proc. Natl. Acad. Sci. U.S.A. 107, 7235-7239 (2010).

46. Y. Nishino et al, Phys. Rev. Lett. 102, 018101 (2009). 
47. C. Song et al, Phys. Rev. Lett. 101, 158101 (2008).

48. H. Jiang et al., Phys. Rev. Lett. 100, 038103 (2008).

49. R. Neutze et al., Nature 406, $752-757$ (2000).

50. T. Ekeberg et al., Phys. Rev. Lett. 114, 098102 (2015).

51. T. Kimura et al., Nat. Commun. 5, 3052 (2014).

52. M. Gallagher-Jones et al., Nat. Commun. 5, 3798 (2014).

53. M. F. Hantke et al., Nat. Photonics 8, 943-949 (2014).

54. H. N. Chapman et al., Nature 470, $73-77$ (2011).

55. J. Tenboer et al., Science 346, 1242-1246 (2014).

56. J. Kern et al., Science 340, 491-495 (2013).

57. W. Liu et al., Science 342, 1521-1524 (2013).

58. A. Barty et al., Nat. Photonics 2, 415-419 (2008).

59. J.-D. Nicolas et al., J. Appl. Cryst. 47, 1596-1605 (2014).

60. J. Miao, K. O. Hodgson, D. Sayre, Proc. Natl. Acad. Sci. U.S.A. 98, $6641-6645$ (2001).

61. R. Fung, V. Shneerson, D. K. Saldin, A. Ourmazd, Nat. Phys. 5, 64-67 (2009).

62. N.-T. D. Loh, V. Elser, Phys. Rev. E 80, 026705 (2009).

\section{Acknowledgements}

We thank C. Pellegrini and J.M. Rodenburg for stimulating discussions, H. Jiang, J. Zhou, Y. Yang and D. Adams for help with the figures and references. J.M. and M.M.M. acknowledge the support by the DARPA PULSE program through a grant from AMRDEC. I.K.R. acknowledges support from an ERC "Advanced" grant.

\section{Figure Legends}


Fig. 1. Schematic layout of five main CDI methods and iterative phase retrieval algorithms. (A) Plane-wave CDI. (B) Bragg CDI. (C) Ptychographic CDI. (D) Fresnel CDI. (E) Reflection CDI. (F) A common feature of phase retrieval algorithms iterating between real and reciprocal space.

Fig. 2. Revolution in coherent $x$-ray sources. (A) The $\mathrm{x}$-ray brilliance of coherent light sources has improved by 20 orders of magnitude in 6 decades (brilliance is a measure of coherent x-ray flux). The inset shows the SASE process to produce extremely intense and short XFEL pulses. (B) Progress in tabletop coherent bandwidth and pulse duration as a function of year. Coherent high harmonics can generate an x-ray super continuum or a series of narrowband peaks spanning the UV to the keV region, supporting the shortest bursts of light to date. The vertical lines show both the transform-limited pulse duration and the chirped isolated bursts that emerge when HHG is driven by infrared lasers. The inset shows the HHG spectrum and laser-like beam.

Fig. 3. Physical science applications of diffractive imaging methods with coherent x-rays. (A) 3D morphology of Fe-rich and Fe-S phases in an olivine matrix (35). (B) Histogram of the Fe-rich phase, Fe-S phase and olivine distribution within a dotted line cube in (A). (C) Phase of the ptychographic reconstruction of a partially delithiated nanoplate of $\mathrm{LiFePO}_{4}$ (38). (D) Chemical composition image of the nanoplate showing the distribution of two chemical species: $\mathrm{LiFePO}_{4}$ and $\mathrm{FePO}_{4}$. (E) 3D imaging of lattice dynamics in a gold nanocrystal (17). Orthogonal cut through the center of the nanocrystal showing the displacement as a function of delay time at three different directions. The $\mathbf{Q}$ vector represents the direction of the displacement field. (F) 3D profile of a ptychographic reconstruction with spatial resolution $<1.3 \lambda$ horizontally and sub-nm vertically, which was acquired using a tabletop HHG source with $<1$ min exposure time (29). 
Fig. 4. Biological applications of diffractive imaging methods with coherent X-rays. (A) 3D mass density distribution of a whole, unstained yeast spore cell, showing nucleus (orange), ER (green), vacuole (white), mitochondria (blue), and granules (light blue) (44). (B) 3D image of an unstained human chromosome where the highest electron density is around the centromere (in red) (46). (C) First coherent X-ray diffraction pattern measured from a single, unstained herpesvirus virion and its reconstructed structure (inset), where the capsid is in yellow (47). (D) Quantitative 3D measurements of the osteocyte lacunae (L) and the connecting canaliculi (C) in a bone matrix (15). (E) Two representative diffraction patterns of individual mimivirus particles collected with single LCLS pulses and the corresponding reconstructed structures (insets) (16). (F) Coherent X-ray diffraction pattern collected from a photosystem I nanocrystal using the LCLS, where the interference pattern between the Bragg peaks is visible due to the finite size of the nanocrystal (54). Inset, real space image of the nanocrystal reconstructed from the circled Bragg shape transform. (G) $2 m F_{o}-D F_{c}$ electron density map at $1.0 \sigma$ (purple mesh), obtained from diffraction intensities with more than 15,000 photosystem I nanocrystals (the refined model shown in yellow). 


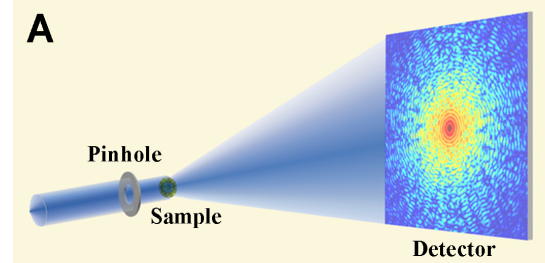

D

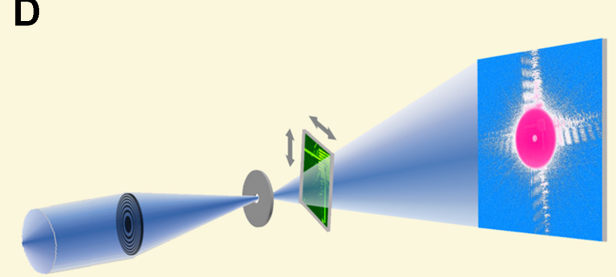

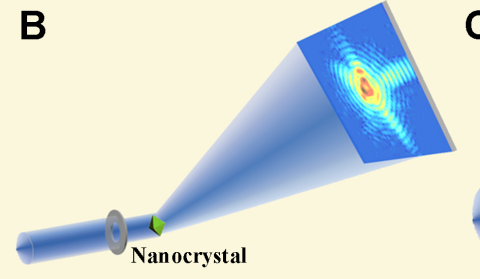

E

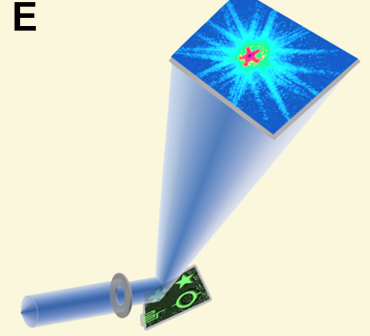

FIG. 1
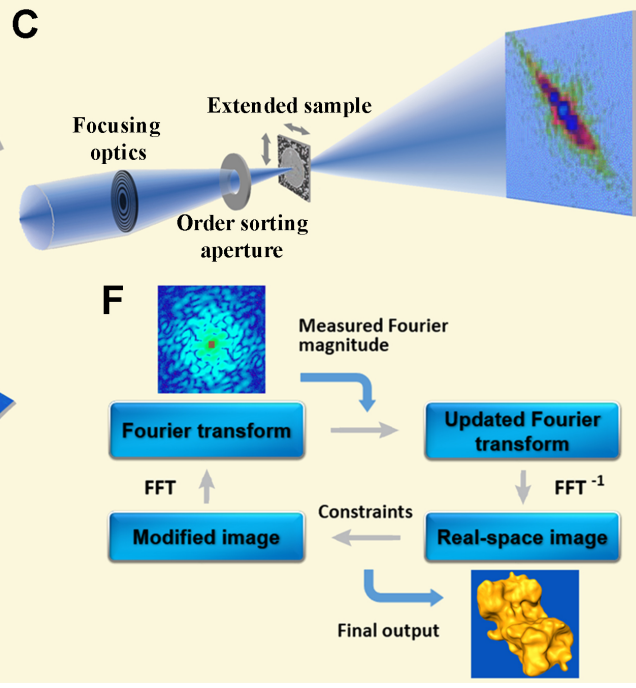

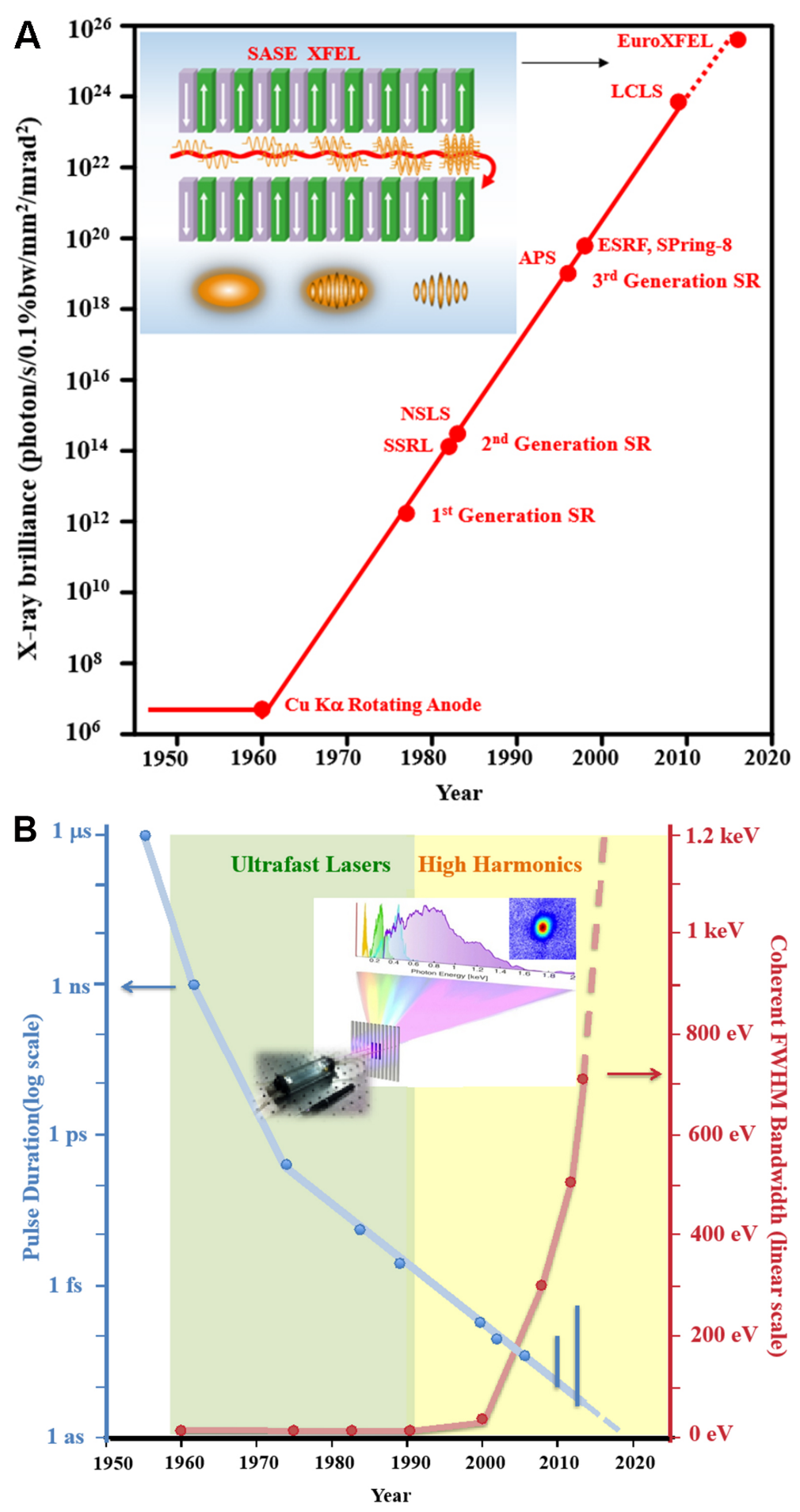

FIG. 2 

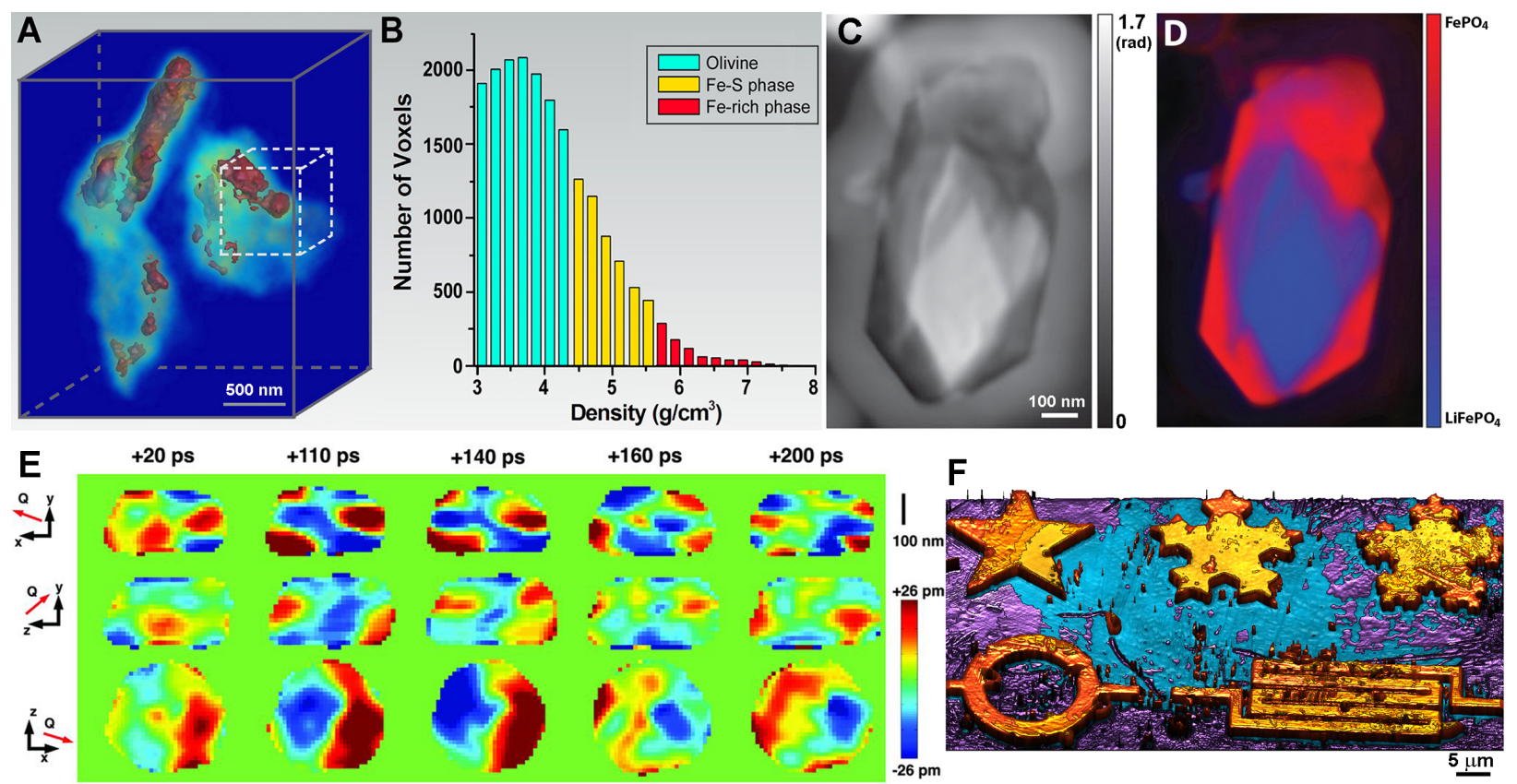

FIG. 3
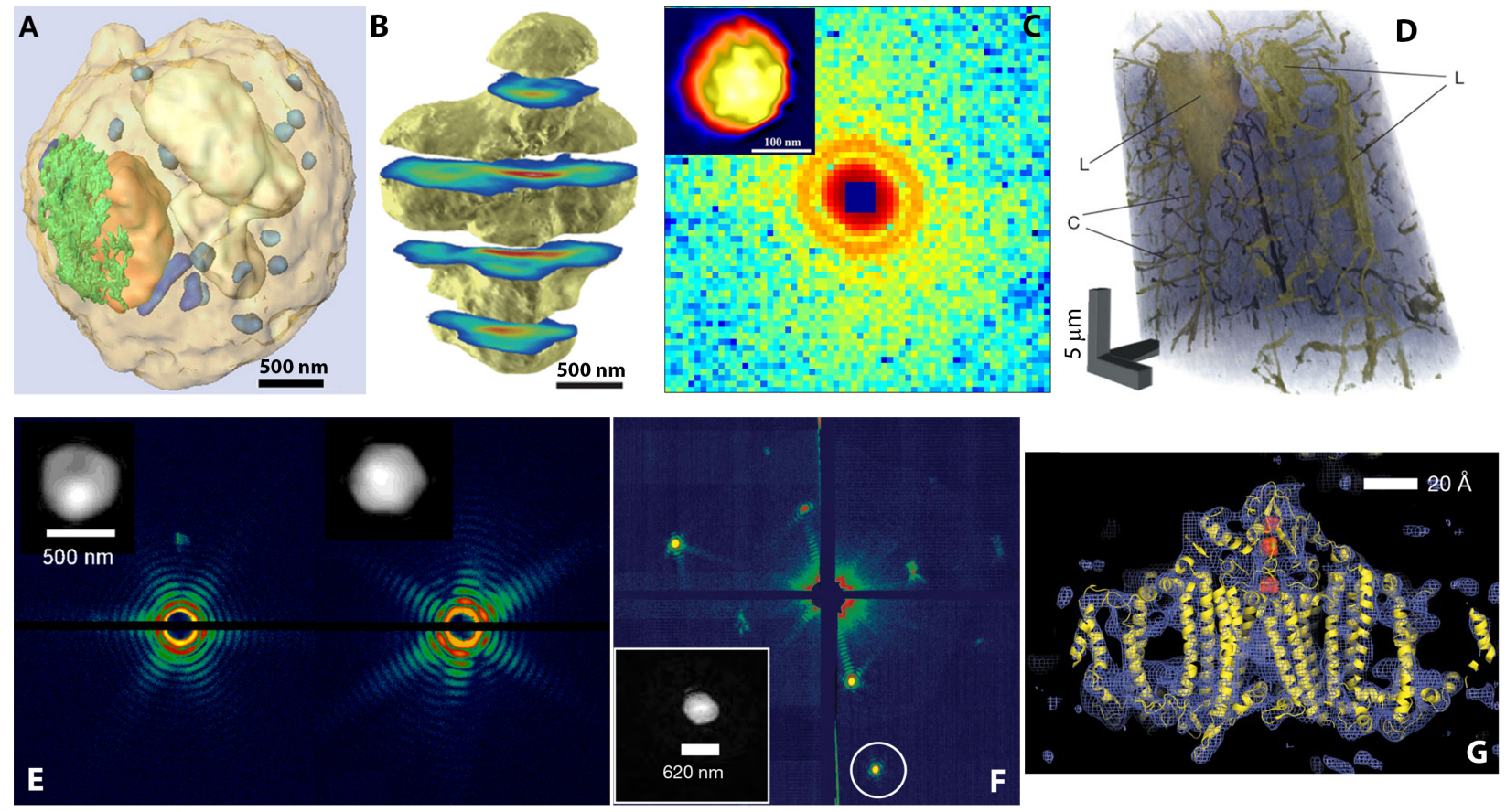

FIG. 4 